\title{
Orthodontic approach to treatment of skeletal mandibular asymmetry: A case report
}

\author{
Eduardo Franzotti Sant'Anna ${ }^{1}$, Cláudia Franzotti Sant'Anna ${ }^{2}$ and Mariana Marquezan ${ }^{1,3 *}$ \\ ${ }^{1}$ Department of Pedodontics and Orthodontics, Universidade Federal do Rio de Janeiro, Rio de Janeiro, Brazil \\ ${ }^{2}$ Private practice, Rio de Janeiro, Brazil \\ ${ }^{3}$ Odontologist, Universidade Federal de Santa Maria, Santa Maria, Rio Grande do Sul, Brazil
}

\begin{abstract}
The patient, a 23 year-old man, presented with a dental Class III malocclusion left subdivision, evident facial asymmetry, lower midline shift, mild contraction of the maxillary arch and an anterior cross bite. There was no history of trauma in the face and jaw or TMJ disorder. The patient was successfully treated by orthodontic approach without performing orthognathic surgery. Pretreatment and post treatment records are presented and discussed.
\end{abstract}

\section{Introduction}

Minor facial asymmetry is observed in all individuals and is considered normal. However, some degree of mandibular asymmetry can compromise function and aesthetics. The etiology of the mandibular deviation can be a combination of genetic and environmental factors, such as syndromes, hypoplasia or hyperplasia of the condyle, fracture and trauma, infection, inflammatory arthritis or unilateral functional cross bite [1].

Unilateral posterior crossbites with mandibular shift should be intercepted in early dentition to avoid asymmetric growth of the jaw [2]. There have been reports of facial symmetry being reestablished after maxillary expansion [3-5]. However, after the growth is complete, TMJs suffer adaptive remodeling and a functional shift of the mandible can become a skeletal asymmetry. The degree of the asymmetry [2], and the patient's perception of it, as well as his/her desire and expectations will guide the decision between opting for surgical correction or orthodontic camouflage.

This case report shows the orthodontic treatment of an adult male with facial asymmetry, who sought improvement in his smile and occlusion, but did not want to proceed with a surgical intervention.

\section{Case report}

\section{Diagnosis and etiology}

The patient, a 23 year old male, with a dental Class III malocclusion, left subdivision, evident facial asymmetry, with mild contraction of the maxillary arch and anterior cross bite (maxillary canine, mandibular canine and right first premolar) sought treatment at our Dental Office (Figures 1-3). The lower midline was shifted approximately $3 \mathrm{~mm}$ to the right, following the mandibular deviation. He also presented anterior spacing: $3 \mathrm{~mm}$ in the maxillary, and $2 \mathrm{~mm}$ in the mandibular arch. There was no history of trauma in the face or jaw. Although these signs could indicate a functional cross bite with mandibular shift to the right, no difference was observed between centric occlusion and centric relation. There was no mandibular deviation during mouth opening
(Figure 4) and the left condyle was narrower and longer than the right, indicating skeletal asymmetry (Figures 5 and 6). The anteroposterior length of the mandible was normal, and the patient was skeletal Class I - ANB $4^{\circ}$ (Figure 7), without any history of TMJ disorder.

The patient reported an early intervention with removable orthopedic appliance for maxillary expansion when he was 9 years old, which failed to correct the malocclusion. He mentioned he has been asymmetric since he was a child and grew asymmetrically over the years.

\section{Treatment objectives}

Based on the diagnostic records, the retreatment objectives were to (1) improve the asymmetric mandibular position, (2) improve the maxillary arch form, (3) correct the dental cross bite; (4) close intra arch spaces (5) correct the midline deviation.

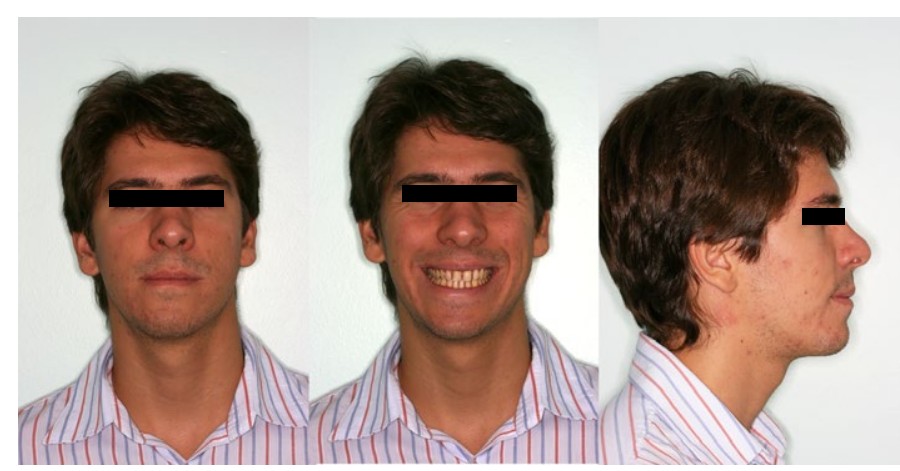

Figure 1. Pretreatment facial photographs.

Correspondence to: Mariana Marquezan, Avenida Professor Rodolpho Paulo Rocco, 325, Ilha do Fundão, Rio de Janeiro, RJ, CEP 21941-617. Tel: 55-2125902727, Fax: 55-21-25909771; E-mail: marianamarquezan@gmail.com

Received: May 01, 2015; Accepted: June 20, 2015; Published: June 24, 2015 


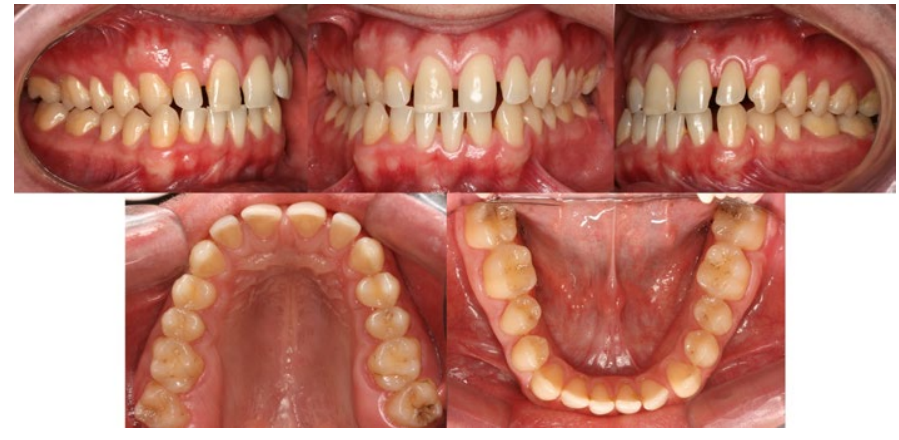

Figure 2. Pretreatment intra-oral photographs.

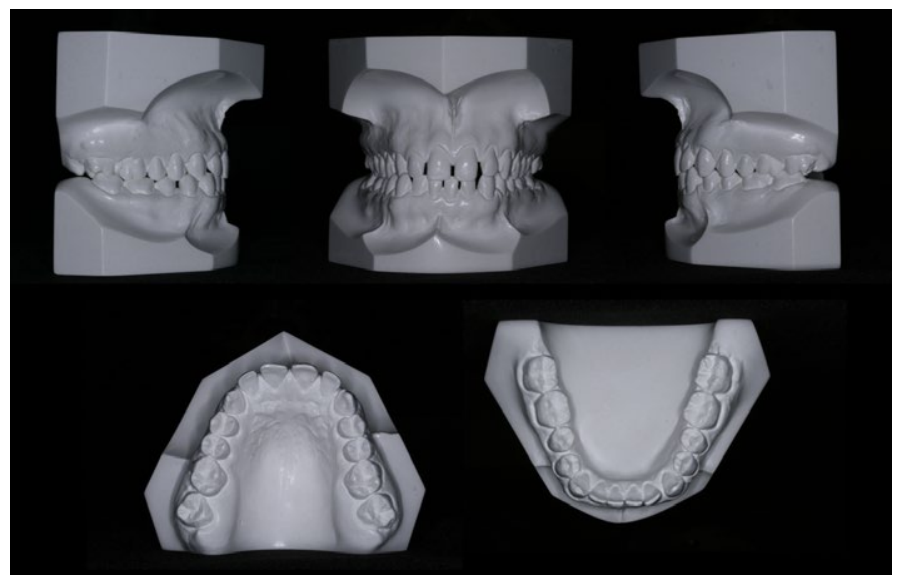

Figure 3. Pretreatment dental casts.

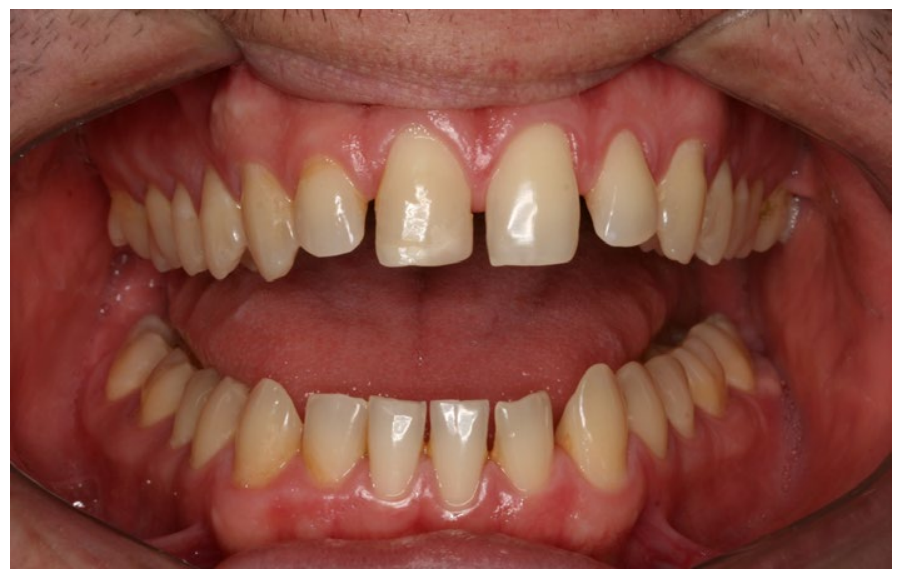

Figure 4. Pretreatment intra-oral photograph during opening mouth.

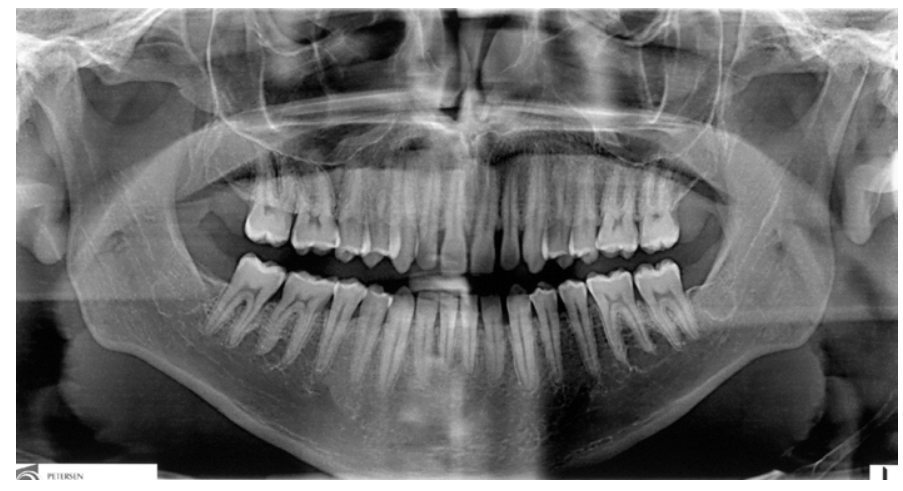

Figure 5. Pretreatment panoramic X-ray.

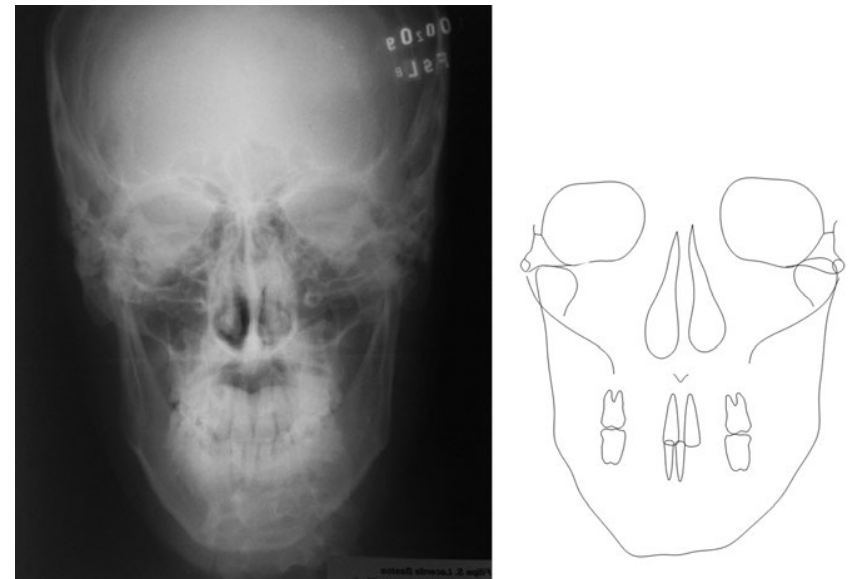

Figure 6. Pretreatment frontal radiograph and cephalometric tracing.
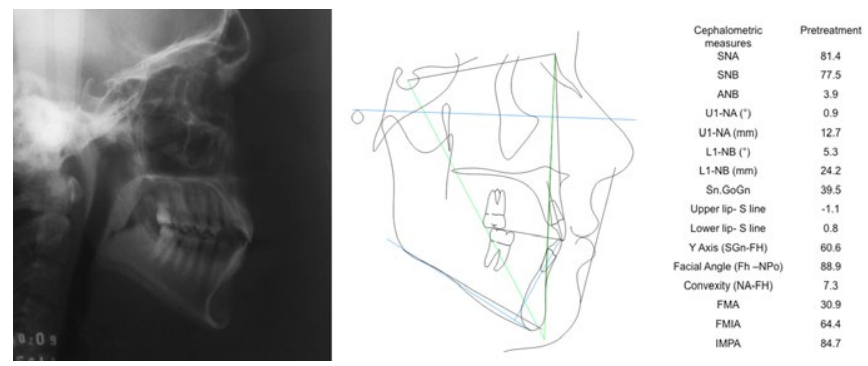

Figure 7. Pretreatment lateral radiograph and cephalometric tracing.

\section{Treatment alternatives}

Treatment options were (1) a combination of orthognathic surgery and orthodontic treatment to address the mandibular and facial asymmetries; or (2) perform a compensatory orthodontic treatment establishing a better occlusion and camouflaging the asymmetry. In this option of treatment, objective 1 and 5 would not be achieved. The patient chose the second option.

\section{Treatment progress}

The orthodontic appliance was inserted/put into place from second molar to second molar, using Edgewise standard brackets (.022x.028" slot). The alignment and leveling started with NiTi .014", stainless steel (SS") $.016 ", .018$ "and .020 ". During the use of the round stainless steel arch wires, $1 / 4$ " cross bite elastic was used on the right side. Midline correction with and elastomeric chain of the mandibular arch started with .020 " archwire with stop bent loops close to the second molar tubes, bringing each tooth to the left side from the mandibular left lateral incisor to the mandibular right second molar. Some mesio-distal stripping was performed in both mandibular lateral incisors.

After this, a .019 x .025" SS maxillary arch wire was installed and the midline correction proceeded using elastometric chain in association with tie together for anchorage. This mechanics was performed from the maxillary right lateral incisor to the maxillary left second molar. Class III 5/16 elastic" was worn on the left side for a period of 6 months to aid the correction of the left sagittal relationship, move the maxillary left first molar mesially and serve as anchorage for the mandibular arch during tooth-to-tooth left correction of the midline 
(Figure 8). Vestibular torque was applied to tooth 13 to promote better intercuspidation.

A .018 x .025 " SS arch wire was inserted in the mandibular arch, which was expanded on the left side, and the maxillary arch was contracted. Differential torques were used on posterior teeth for better intercuspidation: palatal torque of the maxillary right molars and buccal torque of the maxillary left molars; lingual torque of the mandibular right molars and buccal torque of mandibular left molars. Next, diagonal $1 / 4$ " elastic was used between teeth 23 and 43 for 4 months to help the midline correction (Figure 9).

After the active phase of the orthodontic treatment was complete, a removal wraparound appliance was put into place in the maxillary arch and a fixed bar was bonded to mandibular anterior teeth and maxillary central incisors.

Patient underwent dental bleaching and restorative dentistry to improve the esthetic appearance of the teeth.

\section{Results}

A good result was achieved with the camouflage treatment. Class I molar and canine relationships, and ideal overjet and overbite were attained. The cross bite was corrected and the maxillary arch presented a better shape. The midline was improved, but a mild deviation remains. Facial aspect was unchanged, since no surgery was performed. However, the aspect of the smile, and consequently of the face, is much better after orthodontic and restorative treatment (Figures 10-12). The post treatment panoramic radiograph shows no root resorption or periodontal bone loss (Figure 13). Lateral and frontal final radiographies show no skeletal change, but improvement of the teeth (Figures 14-18). TMJ remained asymptomatic.

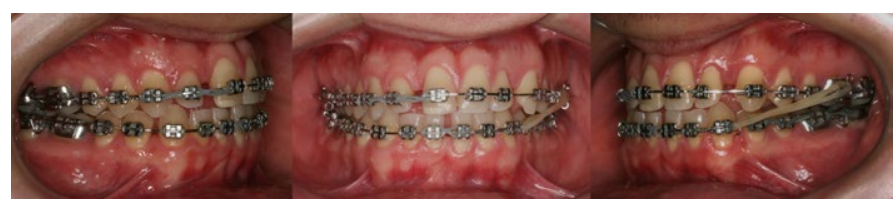

Figure 8. Intra-oral photographs during treatment. Elastomeric chains being used for midline corrections; and Class III 5/16 elastic used for dental sagittal correction.

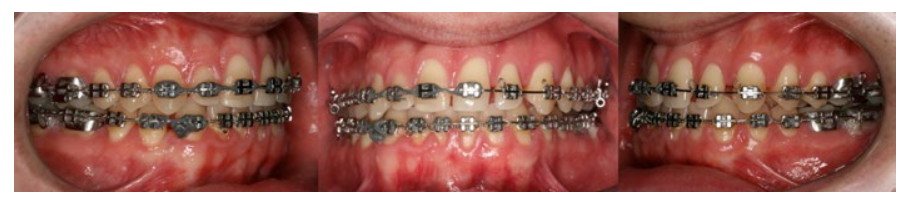

Figure 9. Differential torques applied in posterior region and Kobaiashi hooks placed on teeth 23 and 42 for diagonal $1 / 4$ " elastic used for midline correction.

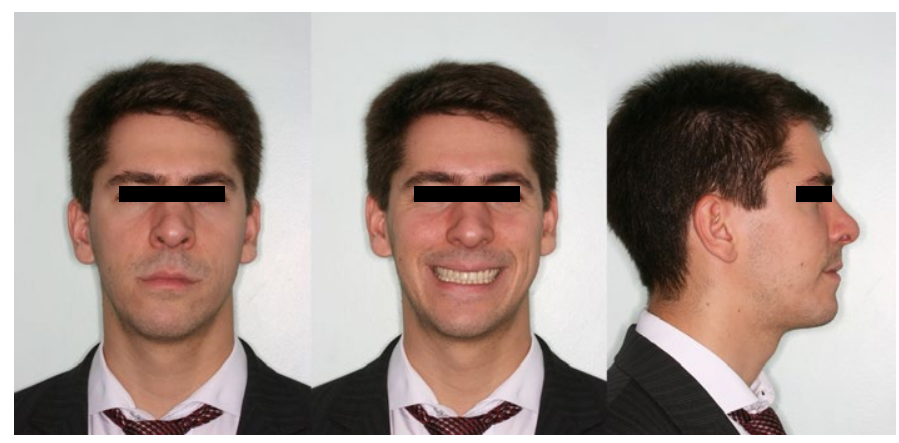

Figure 10. Post treatment facial photographs.

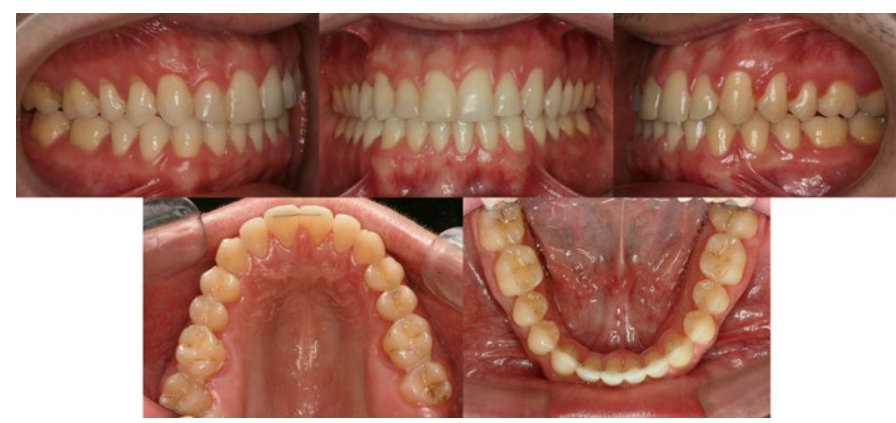

Figure 11. Post treatment intra-oral photographs.

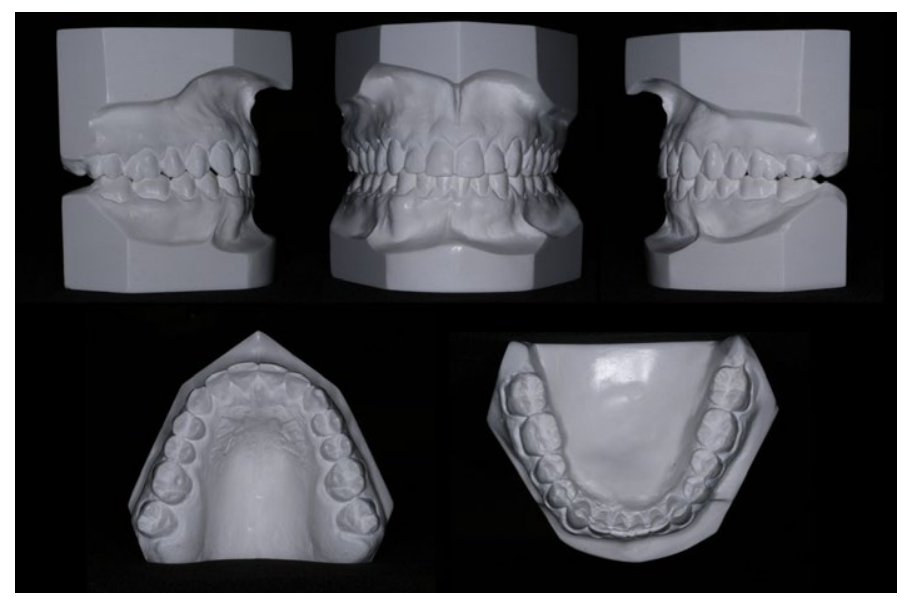

Figure 12. Post treatment dental casts.

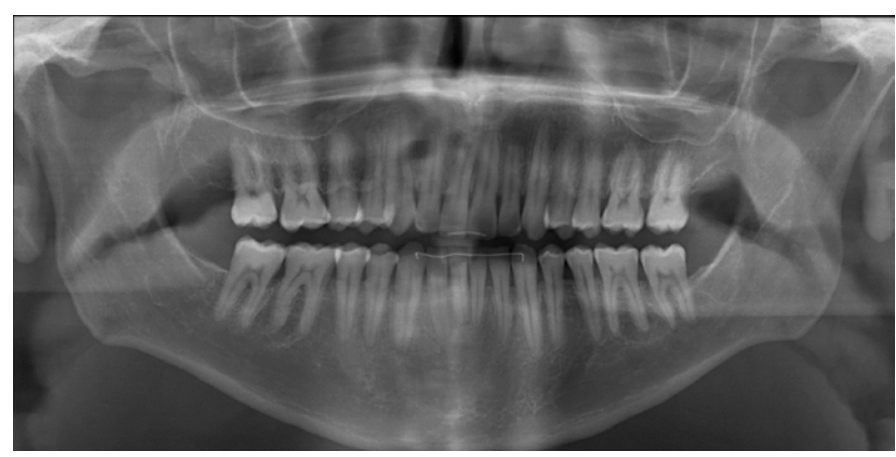

Figure 13. Post treatment panoramic X-ray.

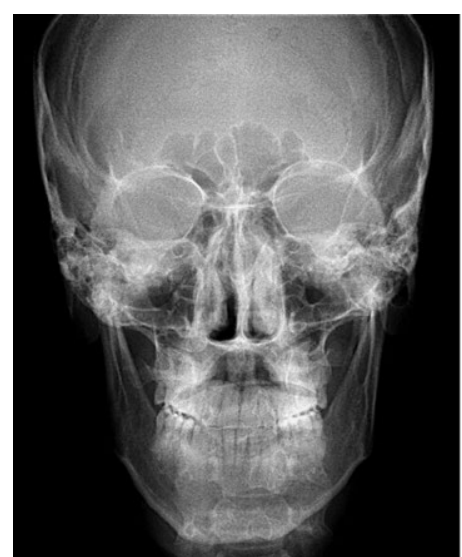

Figure 14. Post treatment frontal radiograph and cephalometric tracing. 

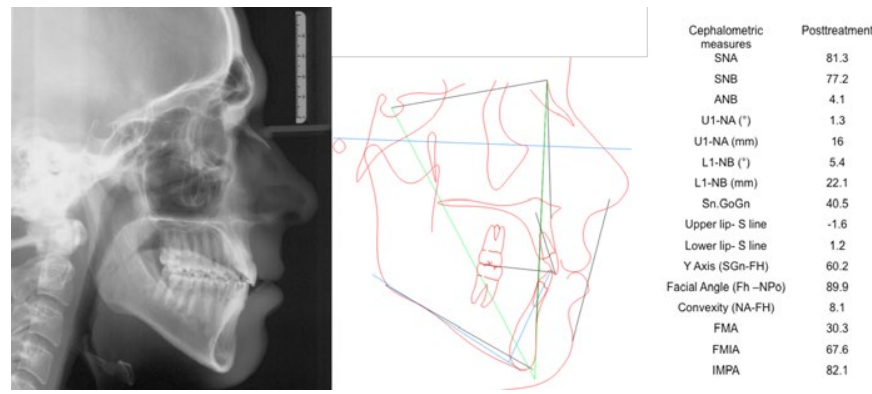

Figure 15. Post treatment lateral radiograph and cephalometric tracing.

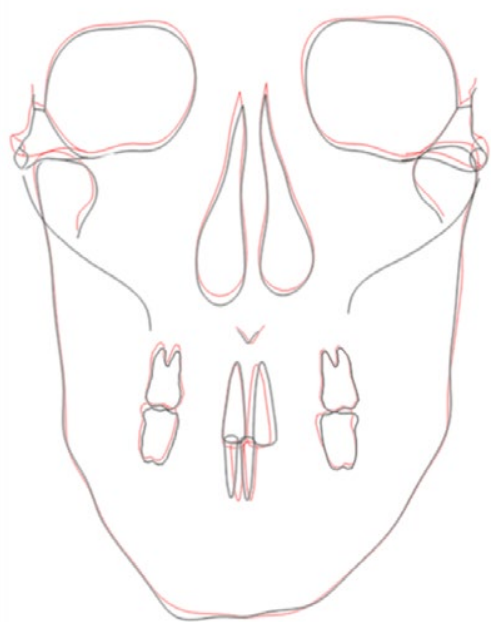

Figure 16. Superimposed pretreatment and post treatment frontal tracings.

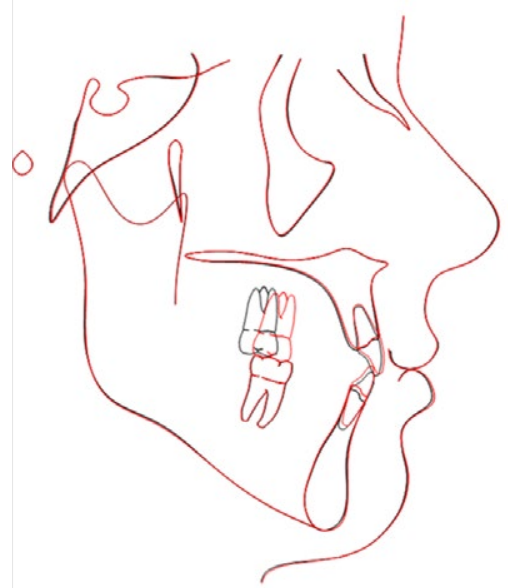

Figure 17. Superimposed pretreatment and post treatment lateral tracings.

After 2 years post treatment, the case is stable and the patient is satisfied with the result (Figures 19 and 20).

\section{Discussion}

Normal faces are not completely symmetric, they consists of a multitude of minor asymmetric components [6]. Perfectly symmetric faces, constructed in computer software, are unnatural and are not as beautiful as the natural asymmetric ones [7]. It is known that a certain

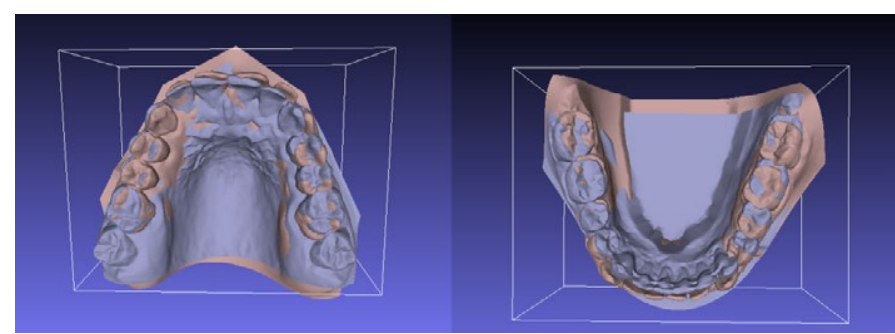

Figure 18. Superimposed pretreatment and post treatment upper and lower plaster casts Superimpositions were realized using the software MeshLab (Italian National Research Council, 2013).

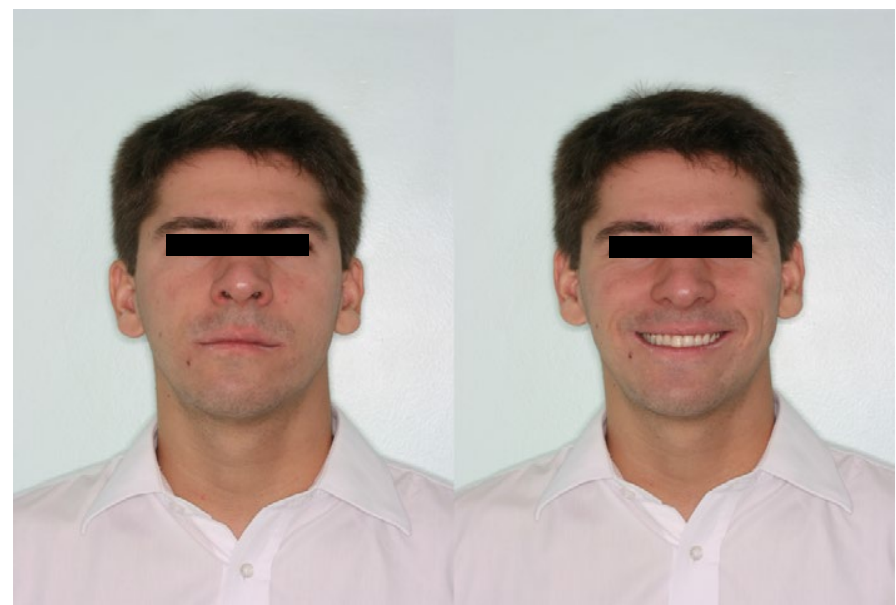

Figure 19. 2-years post treatment up facial photographs.

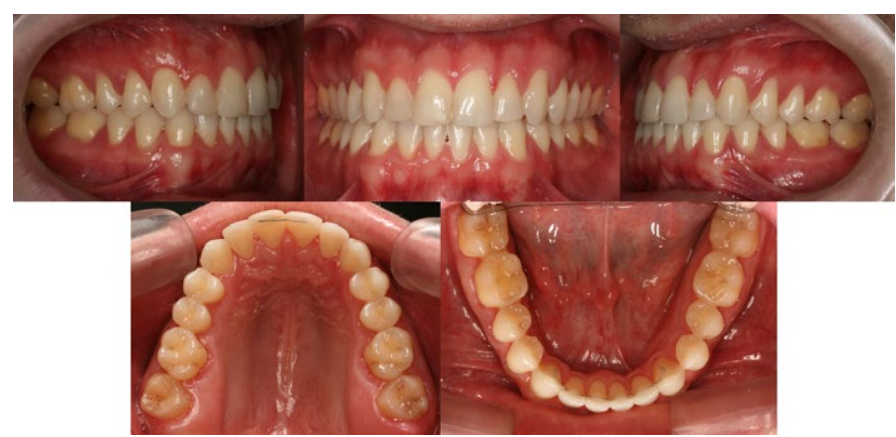

Figure 20. 2-years post treatment up intra-oral photographs.

degree of asymmetry is beautiful, but the border line between normal asymmetry and asymmetry that requires treatment is subjective [8] and varies among professionals and lay people [6]. Because of this, the patient's perception of, and desire to correct or not to correct the asymmetry must be considered.

How patients perceive the severity of their problems is a factor in the decision between camouflage and ortho-surgical treatment. There is good evidence that the more the person perceives herself (or himself) as normal, the more likely he or she is to choose orthodontics alone and to be satisfied with the outcome. Conversely, patients who perceive themselves as outside the normal range are more likely to prefer surgery and to be dissatisfied with tooth movement alone [9]. Problem severity cannot be evaluated just from cephalometric radiographs, dental casts, and other physical records. The orthodontist cannot set physical characteristics as the sole definition of whether surgical correction is required or whether orthodontics alone would be satisfactory [9]. The patient described in this article sought correction of the occlusion and 
did not want to be submitted to orthognathic surgery. The patient was satisfied with the treatment.

Orthodontic camouflage is a feasible option of treatment for mild skeletal malocclusions. The goal of dental camouflage is to correct the skeletal relationships by orthodontically repositioning the teeth in the jaws, so that there is an acceptable dental occlusion and an aesthetic facial appearance [10]. The treatment of malocclusions involving mandibular lateral asymmetry can be complicated and challenging [11]. There are a few case reports found in the literature. Anhoury [11] showed a successful case of treating a young female patient with asymmetry, with tooth extraction and asymmetric mechanics. Onodera and Celar [12] showed a series of pilot cases, in which asymmetric torques were applied to guide asymmetric mandibles into a more central position, partially correcting the midline shift. In this case report, the patient had some spaces in anterior region, which facilitated improvement of the midline and sagittal correction. The mild asymmetry of the face and arcades allowed the transversal correction using cross bite elastics and applying differential torques in the posterior region to favor intercuspidation.

The current literature does not show comparison of asymmetric patients treated with camouflage or orthognatic surgery. Neither final results nor stability were compared for these cases. Comparisons of orthodontic versus surgical correction of Class II problems in nongrowing patients were already published [9]. Both types of treatment met their treatment objectives and the jaw relationships and dental occlusion were similar at the end of treatment. The amount of change produced by treatment was larger in the surgical groups, and they experienced a component of skeletal change that the orthodontic patients did not. The camouflage group overall was very satisfied with their treatment and had fewer functional and TMJ problems than any surgical group. They were positive about aspects of facial appearance that were not affected by treatment as well as those that were [9]. In this case report, the post treatment aspect exceeded the patient's expectations.

In boarder line cases, when camouflage or orthognatic surgeries are possible options, the ratio of benefit to risk must be considered for each procedure. The important decision is whether the greater improvement in dentofacial image, that is possible with surgery, would be worth the increased cost and risk [9]. The risks of surgery obviously can be much greater than those of a nonsurgical approach. The most common surgical risk is decreased sensation of the lips, whereas the greatest risk for camouflage patients appears to be resorption of the maxillary incisor roots as they are retracted and torqued against the lingual cortical plate [9]. In this case report, no important root resorption was observed in the end of treatment.

After orthodontic treatment, the patient's facial asymmetry remained the same, and the relationship between the condyles and temporal fossae were probably not significantly changed. The patient's TMJ remained asymptomatic during and after orthodontic treatment. The result is in accordance with previous studies, which reported that orthodontic treatment is essentially neutral as regards the TMJ, and is not a risk factor for developing TMD [13], even when anatomical changes are observed in TMJ. However, more specific studies are needed to document condylar position changes in the fossa as a result of unilateral posterior cross bite correction in adults, by orthodontic means alone [11].

Considering long-term stability, it was already published that for correction of Class II problems in non-growing patients, both orthodontic and surgical approaches showed similar correction of the malocclusion, although the camouflage group had slightly greater overjet at 1 year post-treatment [14]. In this case report, after a twoyear follow-up, the case was stable and the patient was happy with the result.

\section{Conclusion}

Successful management of the Class III malocclusion with unilateral cross bite was achieved by orthodontic treatment. The facial asymmetry remained the same, however the patient was happy with the aesthetic improvement acquired with his new smile. The decision for opting between orthognathic surgery and orthodontic camouflage depends on the degree of mandibular asymmetry and the patient's wishes.

\section{Acknowledgement}

We thank Andreia Silva Moura dos Santos for her assistance and help with the clinical pictures throughout the treatment and Daniel Brunetto for his assistance with the cephalometric tracings.

\section{References}

1. Pirttiniemi PM (1994) Associations of mandibular and facial asymmetries--a review. Am J Orthod Dentofacial Orthop 106: 191-200. [Crossref]

2. Bishara SE, Burkey PS, Kharouf JG (1994) Dental and facial asymmetries: a review. Angle Orthod 64: 89-98. [Crossref]

3. Pinto AS, Buschang PH, Throckmorton GS, Chen P (2001) Morphological and positional asymmetries of young children with functional unilateral posterior crossbite. Am J Orthod Dentofacial Orthop 120: 513-520. [Crossref]

4. Primozic J, Ovsenik M, Richmond S, Kau CH, Zhurov A (2009) Early crossbite correction: a three-dimensional evaluation. Eur J Orthod 31: 352-356. [Crossref]

5. Kwak YY, Jang I, Choi DS, Cha BK (2014) Functional evaluation of orthopedic and orthodontic treatment in a patient with unilateral posterior crossbite and facial asymmetry. Korean J Orthod 44: 143-153. [Crossref]

6. Edler R, Wertheim D, Greenhill D (2001) Clinical and computerized assessment of mandibular asymmetry. Eur J Orthod 23: 485-494. [Crossref]

7. Haraguchi S, Iguchi Y, Takada K (2008) Asymmetry of the face in orthodontic patients Angle Orthod 78: 421-426. [Crossref]

8. Lee MS, Chung DH, Lee JW, Cha KS (2010) Assessing soft-tissue characteristics of facial asymmetry with photographs. Am J Orthod Dentofacial Orthop 138: 23-31. [Crossref]

9. Mihalik CA, Proffit WR, Phillips C (2003) Long-term follow-up of Class II adults treated with orthodontic camouflage: a comparison with orthognathic surgery outcomes. Am J Orthod Dentofacial Orthop 123: 266-278. [Crossref]

10. Naragond A, Kenganal S, Sagarkar R, Sugaradday (2013) Orthodontic Camouflage Treatment in an Adult Patient with a Class II, Division 1 Malocclusion - A Case Report. J Clin Diagn Res 7: 395-400. [Crossref]

11. Anhoury PS (2009) Nonsurgical treatment of an adult with mandibular asymmetry and unilateral posterior crossbite. Am J Orthod Dentofacial Orthop 135: 118-126. [Crossref]

12. Onodera K, Celar A (2012) Midline correction by asymmetric reciprocal torque: a pilot study. J Orofac Orthop 73: 317-325. [Crossref]

13. Sadowsky C (1992) The risk of orthodontic treatment for producing temporomandibular mandibular disorders: a literature overview. Am J Orthod Dentofacial Orthop 101: 7983. [Crossref]

14. Proffit WR, Phillips C, Douvartzidis N (1992) A comparison of outcomes of orthodontic and surgical-orthodontic treatment of Class II malocclusion in adults. Am J Orthod Dentofacial Orthop 101: 556-565. [Crossref]

Copyright: (C2015 Sant'Anna EF. This is an open-access article distributed under the terms of the Creative Commons Attribution License, which permits unrestricted use, distribution, and reproduction in any medium, provided the original author and source are credited. 